\title{
Reflets
}

Revue ontaroise d'intervention sociale et communautaire

\section{Sourds ou malentendants, une question de culture?}

\section{Marc Charron}

Volume 4, numéro 2, automne 1998

Personnes vivant avec une incapacité

URI : https://id.erudit.org/iderudit/026218ar

DOI : https://doi.org/10.7202/026218ar

Aller au sommaire du numéro

Éditeur(s)

Reflets : Revue ontaroise d'intervention sociale et communautaire

ISSN

1203-4576 (imprimé)

1712-8498 (numérique)

Découvrir la revue

Citer cet article

Charron, M. (1998). Sourds ou malentendants, une question de culture? Reflets, 4(2), 63-86. https://doi.org/10.7202/026218ar

Tous droits réservés (C) Reflets : Revue ontaroise d'intervention sociale et communautaire, 1998
Ce document est protégé par la loi sur le droit d'auteur. L’utilisation des services d’Érudit (y compris la reproduction) est assujettie à sa politique d'utilisation que vous pouvez consulter en ligne.

https://apropos.erudit.org/fr/usagers/politique-dutilisation/ 


\section{Sourds ou malentendants, une question de culture?}

\section{Marc Charron ${ }^{1}$}

Chargé de cours, Université Laurentienne

Déficient auditif, on l'est naturellement. C'est physique. Sourd, on le devient, c'est social. Cela s'apprend. Cela se prend. Cela s'attrape auprès des siens : pairs, aînés, adultes Sourds.

(Mottez 1985: 13)

\section{Introduction}

Cet exergue a guidé la présente recherche-action sur les Sourds ${ }^{2}$. Celle-ci cherche à comprendre la culture sourde et à connaitre le degré d'adhésion des Sourds à celle-ci. En voulant explorer la culture sourde, on rejetait d'emblée le biais d'un savoir médical constitué sur la surdité, qui réduit le fait d'être sourd au nombre de décibels qu'un individu peut ou ne peut pas entendre et à évaluer ses effets physiologiques et psychologiques. En effet, la médicalisation de la surdité, de façon subtile, opère un double processus. D'une part, elle infériorise le Sourd sur la base du modèle physiologique de l'infirmité qui nécessite l'intervention des entendants dans la vie des Sourds. D'autre part, «elle vise aussi à anéantir la prétention de la communauté sourde à jouir du statut de minorité linguistique et culturelle en droit d'assumer ellemême son présent et son avenir» (Lane 1994 : 49). C'est donc ce droit à l'existence d'une culture sourde autonome que nous avons voulu saisir. La langue, la façon de communiquer seront au centre de cette recherche. 
Cette voie est apparue difficile surtout lorsqu'il s'est agi de constituer un savoir sur les Sourds, tout en étant conscient du danger que comporte l'institution audiste qui a balisé toute démarche de connaissances sur les Sourds jusqu'à présent. L'audisme est:

Un système institutionnel chargé de s'occuper des sourds, c'est-à-dire de s'en occuper en émettant des affirmations à leur sujet, en autorisant des jugements sur eux, en les décrivant, en enseignant sur leur cas, en dictant où ils vont à l'école et, parfois, où ils habitent. Bref, l'audisme est le moyen que se sont donnés les entendants pour dominer, restructurer et commander la communauté sourde (Lane 1994 : 46).

Cette institution audiste est omniprésente lorsqu'on aborde l'étude de la communauté sourde. Et il semble impossible d'y échapper. En recherche, cela soulève la question suivante, formulée par Lane : «Comment pouvons-nous protéger la recherche sur les sourds du paternalisme structurel qui régit la formation de professionnels, le financement de leurs travaux, leurs accès aux sujets, la publication de leurs résultats, etc.?» (Lane 1994 : 54).

Cette question éthique ne demeure pas sans réponse. En effet, pour Lane, la solution la plus efficace :

consisterait à faire participer les Sourds eux-mêmes à tous les niveaux de l'édifice. Nous devons établir une stratégie de recrutement et de formation d'un nombre bien plus élevé de directeurs de projets de recherche sourds. Nous devons nous adresser en priorité à la communauté sourde afin de trouver des conseillers et des collaborateurs pour concevoir et réaliser des recherches ainsi que des assistants en matière de collecte et d'analyse des données. Enfin, nous devons nous faire guider par elle dans l'interprétation des données (Lane 1994: 54).

Traduit en terme méthodologique, cet objectif signifie de faire une recherche-action avec la communauté sourde. 


\section{Méthodologie}

"...la communauté sourde s'est impliquée dans toutes les étapes initiales de la recherche.»
Pour ce faire, la communauté sourde s'est impliquée dans toutes les étapes initiales de la recherche. Ainsi, la communauté sourde du Nord-est ontarien a déterminé les principaux aspects que devait comporter ce sondage. Le choix du sondage comme méthode d'enquête est important, car on désirait élaborer un portrait quantitatif des besoins des Sourds du Nord-est, afin de faire les représentations politiques qui s'imposent par la suite.

Il a donc été collectivement déterminé, lors de discussions avec l'Association des Sourdes et des Sourds francophones de Sudbury (ASFS), que ce sondage devait porter sur les aspects suivants :

1) l'identification à la communauté sourde;

2) les moyens de communication qu'emploie la communauté sourde dans ses relations avec les entendants et avec les sourds;

3) les services gouvernementaux (justice, médecine, assistance technique et professionnelle de tous genres, etc.) offerts à la communauté sourde.

L'identification avait comme objectif de constituer un répertoire des personnes sourdes ou ayant une déficience auditive dans le Nord-est ontarien dans le but d'élargir le nombre de membres de l'ASFS et aussi de constituer une base de sondage pour le questionnaire, c'est-à-dire de repérer les sujets de l'enquête.

La première étape fut menée par les Sourds qui ont dû multiplier les approches afin d'identifier les personnes atteintes de surdité ${ }^{3}$. Celles-ci ont permis d'identifier 375 personnes sourdes ou malentendantes qui constituent le répertoire des Sourds du Nord-est ontarien.

Par la suite, nous avons fait parvenir un questionnaire et une invitation à assister à des soirées d'animation en Langue des signes québécoise (LSQ), organisées dans les quatre secteurs du Nordest ontarien. Ces secteurs étaient Algoma, Timmins- 
"Or, la communication en LSQ était cruciale à la tenue de cette enquête, car elle permettait l'expression de cette culture sourde. De plus, certains Sourds maîtrisent difficilement le français, oral ou écrit, et l'imposition du français aurait constitué une nouvelle marque de cette culture audiste.»
Témiskamingue, Cochrane et Sudbury-Nipissing. Ces soirées d'animation avaient pour objectif d'offrir aux Sourds des services en LSQ pour faciliter la compréhension du questionnaire et, ainsi, mieux parvenir à cerner leurs besoins.

Les soirées d'animation se sont imposées d'elles-mêmes, compte tenu qu'il était impossible d'interviewer individuellement chacune des personnes en LSQ. Or, la communication en LSQ était cruciale à la tenue de cette enquête, car elle permettait l'expression de cette culture sourde. De plus, certains Sourds maîtrisent difficilement le français, oral ou écrit, et l'imposition du français aurait constitué une nouvelle marque de cette culture audiste.

Cette méthode a ses inconvénients et ses avantages. Elle constitue une méthode d'échantillonnage non probabiliste, ce qui signifie que les résultats de l'échantillon ne peuvent pas être généralisés à l'ensemble de la population sourde ou malentendante du Nord-est ontarien. De fait, cette méthode s'apparente à un échantillon de volontaires, c'est-à-dire qu'elle souffre d'un biais d'auto sélection, car le volontaire est généralement plus positif envers la recherche que ne l'est l'ensemble de la population. Elle a cependant l'avantage de permettre d'atteindre des souspopulations minoritaires en économisant les frais d'un échantillonnage probabiliste ${ }^{4}$.

Le questionnaire comptait trois sections. La première portait sur les services gouvernementaux offerts à la population sourde et, notamment, les services d'interprétation. La seconde abordait l'éducation, l'alphabétisation et la langue. La troisième correspondait aux renseignements socio-démographiques. Ces thèmes correspondent aux trois objectifs définis initialement.

Ce questionnaire a permis de recueillir des données quantitatives sur les besoins de la communauté Sourde, données qui n'existaient pas sur les Sourds francophones du Nord-est ontarien. L'analyse et l'interprétation des données ont été réalisées par le groupe de travail. 


\section{Description de l'échantillon}

«Si nous pouvons

déplorer le peu de

personnes rejointes (91 au total), il faut dire que cette tâche comportait un double défi, celui de prendre contact avec les Sourds et de les sortir de leur anonymat si caractéristique et, plus encore, de les convaincre de participer à une enquête.»
Lors de notre tournée régionale, nous avons rencontré, au total, 91 personnes. Parmi elles, 49 personnes se disaient Sourdes (53,8 $\%)$ et 42 personnes se disaient malentendantes (46,1\%). Il est important de préciser qu'une personne qui se dit Sourde s'affiche comme appartenant à la culture Sourde et revendique cette appartenance. Cela n'a rien à voir avec le degré de surdité ou le niveau de décibels qu'un individu peut ou ne peut pas entendre. La personne elle-même détermine son groupe d'appartenance. De la même façon, un individu peut se dire malentendant et être atteint de surdité. Nous procéderons à l'analyse des résultats en fonction de chacun de ces sous-groupes et nous tenterons d'établir une comparaison entre les Sourds et les malentendants.

Si nous pouvons déplorer le peu de personnes rejointes ( 91 au total), il faut dire que cette tâche comportait un double défi, celui de prendre contact avec les Sourds et de les sortir de leur anonymat si caractéristique et, plus encore, de les convaincre de participer à une enquête. En effet, l'exclusion dont les Sourds sont l'objet rendait cette tâche d'autant plus complexe.

\section{Sourds et Sourdes}

Parmi les Sourds (49 personnes au total), la majorité se réclame de la culture Sourde francophone, (45 personnes).Trois personnes disent être Sourdes bilingues et une autre Sourde anglophone. Cette double identification à la culture Sourde et à la culture francophone est importante, car l'enquête visait à mieux connaitre ce groupe quasi invisible dans la société ontarienne.

Les Sourds ayant participé à l'enquête sont majoritairement de sexe masculin (47\%), alors que $37 \%$ sont de sexe féminin. Or, il est étonnant de constater qu'à une question aussi usuelle que d'identifier son sexe, $16 \%$ des répondants et répondantes n'ont 
pas répondu. Ce résultat surprenant peut se comprendre.Au niveau du pré-test, certaines personnes avaient déjà souligné le fait que de connaître le sexe d'une personne pouvait mener à une identification qu'elle ne désirait pas. Qui plus est, la communauté Sourde est fermée sur elle-même. Le risque d'être reconnu, dans un réseau tissé aussi serré, est perçu comme un danger réel.Aussi, ne faut-il pas se surprendre d'avoir un nombre de non-réponses élevées. D’autres éléments démontrent aussi cette sensibilité des Sourds à une identification trop personnelle ou à une invasion de leur vie privée, comme on le verra plus loin.

Le tableau 1 dresse le portrait de l'échantillon des Sourds de l'enquête.

Tableau 1

\section{Âge, état civil et apprentissage de la surdité des répondants Sourds de l'enquête}

\begin{tabular}{|ll|lr|ll|}
\hline \multicolumn{2}{|c|}{ Âge (N=46) } & \multicolumn{2}{|c|}{ État civil (N=46) } & \multicolumn{2}{c|}{$\begin{array}{c}\text { Apparition de la surdité } \\
\text { (N=46) }\end{array}$} \\
\hline Moins de 24 ans & $24 \%$ & Mariés & $63 \%$ & Avant l'âge de 5 ans $\quad 9 \%$ \\
\hline 25 à 44 ans & $24 \%$ & Union libre & $9 \%$ & Entre 5 ans et 14 ans $\quad 18 \%$ \\
\hline 45 à 64 ans & $9 \%$ & Célibataire & $26 \%$ & Entre 15 et 19 ans $\quad 2 \%$ \\
\hline 65 ans et plus & $13 \%$ & Veuf ou veuve & $2 \%$ & Après 40 ans & $11 \%$ \\
\hline
\end{tabular}

«La surdité chez les Sourds devient synonyme d'une appartenance collective."
L'appartenance à la culture Sourde favorise également l'épanouissement de leur culture francophone, l'un n'allant pas sans l'autre. D'ailleurs, lorsqu'on demande au répondant à quelle culture il s'identifie, $83 \%$ répondent appartenir à la culture sourde francophone, tandis que $13 \%$ s'identifient à la culture francophone entendante et, finalement, un autre $3 \%$ à la culture anglophone entendante. La surdité chez les Sourds devient synonyme d'une appartenance collective.

La majorité des Sourds ont un père entendant $(87 \%)$ et une mère entendante (95\%). La surdité n’est donc pas héréditaire. Seulement $2 \%$ de notre échantillon ont respectivement un père ou une mère sourde. Les autres répondants mentionnent que leurs 
«Si le besoin d'interprète se fait sentir, les Sourds privilégieront les membres de leurs familles, eux qui ont agi d'intermédiaires depuis leur enfance. Cette utilisation n'est d'ailleurs pas l'expression d'une volonté manifeste, car, plus souvent qu'autrement, elle vise à pallier le manque de disponibilité d'interprète professionnel.» pères $(11 \%)$ ou leurs mères $(3 \%)$ sont malentendants ou malentendantes.

La langue maternelle du père (79\%) et de la mère (91\%) est le français. Les Sourds et les Sourdes sont donc parties prenantes de la culture reçue en héritage de leurs parents. L'attachement à la culture francophone se manifeste également dans la langue d'usage. Si $45 \%$ des répondants utilisent le français, $49 \%$ utilisent la LSQ comme langue d'usage. Plus que le français, l'identification à la culture sourde passe par l'emploi de la LSQ dans les contacts quotidiens avec les autres Sourds ou avec la famille. Seulement $6 \%$ des répondants utilisent l'anglais comme langue d'usage.

Si la surdité n'a rien d'héréditaire, il importe de voir qu'elle force la solidarité familiale en développant une interdépendance beaucoup plus grande entre les membres de la famille. Ceux-ci sont alors appelés à agir d'intermédiaires entre l'univers des Sourds et le monde des entendants.

Cela apparaît dans les données recueillies à une question à réponses multiples portant sur les moyens les plus fréquemment utilisés par les Sourds pour communiquer avec un entendant. En effet, si les Sourds emploient le plus souvent une combinaison de signes, de lecture labiale et de français dans une proportion de $55 \%$, le recours à un membre de la famille servant d'interprète (45\%), le recours à un interprète professionnel (30\%) ou un ami interprète (17 \%) suivent dans l'ordre.

Cette gradation des moyens est représentative des Sourds.Avant tout, elle montre l'autonomie des Sourds qui se débrouillent tant bien que mal dans un monde d'entendants, sans recourir à aucune aide externe. Beaucoup de Sourds parviennent, par la méthode oraliste, à communiquer avec le milieu environnant en français ou en anglais parlé. Si le besoin d'interprète se fait sentir, les Sourds privilégieront les membres de leurs familles, eux qui ont agi d'intermédiaires depuis leur enfance. Cette utilisation n'est d'ailleurs pas l'expression d'une volonté manifeste, car, plus souvent qu'autrement, elle vise à pallier le manque de disponibilité d'interprète professionnel.

Le caractère francophone de leur communication avec un entendant est remarquable. En effet, $58 \%$ utilisent le français parlé 
ou écrit pour communiquer avec un entendant, même dans un contexte majoritairement anglophone comme le Nord-est de l'Ontario. La raison tient au fait que les Sourds évoluent dans un milieu relativement clos sur lui-même, celui de la famille. Or, la majorité des familles sont endogames francophones et, même lorsqu'ils se marient, les Sourds épousent une personne Sourde francophone, ce qui favorise la reproduction du fait français.

Au moment du sondage, environ la moitié des Sourds occupent un emploi alors que l'autre moitié ne travaillait pas comme en fait foi le tableau suivant.

Tableau 2

Occupations, domaines d'emploi et paiements de transferts des Sourds occupant un emploi

\begin{tabular}{|ll|ll|ll|}
\hline \multicolumn{2}{|c|}{ Occupation (N=345 ) } & \multicolumn{2}{c|}{$\begin{array}{l}\text { Domaine d'emploi des } \\
\text { sourds (14 sur 17) }\end{array}$} & $\begin{array}{c}\text { Types de paiements de } \\
\text { transferts reçus par les } \\
\text { Sourds sans emploi (N= 15) }\end{array}$ \\
\hline Emploi à temps plein & $50,0 \%$ & Éducation & $4 / 14$ & Pension vieillesse & $33,3 \%$ \\
Emploi à temps partiel & $5,9 \%$ & Commerce & $4 / 14$ & Pension invalidité & $40,0 \%$ \\
Sans emploi & $44,1 \%$ & Administration & $2 / 14$ & Aide sociale & $13,3 \%$ \\
& & Forêt & $2 / 14$ & Autres & $13,4 \%$ \\
& Assistance dentaire & $2 / 14$ & & \\
& & & & & \\
& & & &
\end{tabular}

"...un regard sur le tableau 3 permet de dire que la majorité des Sourds vivent dans l'extrême pauvreté, puisque $61 \%$ environ ont un revenu inférieur à $20000 \$$ annuellement.»
Le sentiment de précarité s'accroît considérablement lorsque les personnes interrogées doivent déclarer leur revenu annuel. En effet, un regard sur le tableau 3 permet de dire que la majorité des Sourds vivent dans l'extrême pauvreté, puisque $61 \%$ environ ont un revenu inférieur à $20000 \$$ annuellement. Et le revenu du conjoint ne compense pas toujours pour la faiblesse de revenu des répondants ou des répondantes. En effet, la situation financière des Sourds est précaire si l'on considère que près du tiers des conjoints ou conjointes des répondantes ou répondantes ne travaillent pas (12 sur 36). 
Tableau 3

Revenu des personnes sourdes et de leur conjoint

\begin{tabular}{|ll|ll|}
\hline \multicolumn{2}{|l|}{ Revenu des personnes sourdes $(\mathbf{N}=\mathbf{3 3})$} & \multicolumn{2}{l|}{$\begin{array}{l}\text { Revenu du conjoint des personnes } \\
\text { sourdes } \mathbf{( N = 2 4 )}\end{array}$} \\
\hline Moins de $10000 \$$ & $36,4 \%$ & Moins de $10,000 \$$ & $37,5 \%$ \\
$10000 \$$ à $19,999 \$$ & $24,3 \%$ & $10000 \$$ à $19999 \$$ & $8,4 \%$ \\
$20000 \$$ à $39999 \$$ & $30,3 \%$ & 20000 à $39999 \$$ & $45,8 \%$ \\
$40000 \$$ et plus & $9,1 \%$ & 40000 \$ et plus & $8,3 \%$ \\
\hline
\end{tabular}

C'est dire que les répondantes ou les répondants Sourds de notre échantillon ont des revenus généralement faibles et plusieurs vivent sous le seuil de pauvreté pour deux personnes au Canada qui est d'environ $24000 \${ }^{6}$.

\section{Satisfactions et insatisfactions}

La culture Sourde francophone, malgré les tendances lourdes qui l'affectent et son rapprochement à une culture de pauvreté, n'est pas sans compter son lot de satisfaction.Ainsi, les Sourds interrogés se disent satisfaits de leur vie familiale $(95 \%)$, de leurs relations amicales (96\%), de leurs relations avec les entendants (96\%), avec les Sourds francophones (98\%) et les Sourds anglophones (86\%). On peut comprendre un tel résultat en suggérant que les Sourds vivent en constante interdépendance, ce qui fait d'eux des personnes généralement ouvertes aux contacts humains. Cette belle unanimité est rompue lorsqu'il s'agit d'items autres que les relations sociales.

Dire que les Sourds rencontrés sont insatisfaits serait une affirmation trop forte. Disons que leur niveau de satisfaction décroît significativement lorsqu'il s'agit des services offerts aux Sourds. En effet, $58 \%$ seulement des répondants se disent satisfaits de ces services, alors que $42 \%$ se montrent insatisfaits, voire très insatisfaits. 
"...les Sourds ont des attentes réduites à l'égard du gouvernement. Ils ont l'impression de n'y pouvoir rien changer.»
Ce taux d'insatisfaction augmente à $45 \%$ lorsqu'il s'agit d'évaluer les services offerts par les gouvernements. Certes, là encore, $55 \%$ des Sourds expriment leur satisfaction des services offerts par les différents paliers de gouvernement. Même les services d'interprétation en langue des signes, LSQ ou ASL, s'avèrent satisfaisants aux yeux des répondants (62\%), tandis que $38 \%$ expriment leur insatisfaction.

Comment expliquer ce haut niveau de satisfaction des répondants lorsqu'ils sont appelés à se prononcer à cette série d'items? Cette question trouve réponse dans le faible niveau d'attentes des Sourds vis-à-vis des gouvernements ou des agences para-gouvernementales, comme la Société canadienne de l'Ouïe (SCO). Longtemps exclus de la vie publique, invisibles aux yeux des gouvernements où la loi du nombre domine, les Sourds n'ont pas l'habitude de faire des pressions et de revendiquer leurs droits. Reclus, leur univers se confine au cadre familial, aux relations amicales qu'ils nouent. C'est ce milieu qui les supporte et le gouvernement semble un outil bien éloigné de leurs préoccupations quotidiennes. Toutefois, là plus qu'ailleurs, les Sourds manifestent une insatisfaction à l'endroit des gouvernements, mais on est loin d'y découvrir une revendication en règle. De fait, les Sourds ont des attentes réduites à l'égard du gouvernement. Ils ont l'impression de n'y pouvoir rien changer.

La meilleure illustration de ce niveau d'attentes réduites est celle du revenu et de la sensibilisation de la population à leur égard. $\mathrm{Si}$, objectivement, les Sourds ont un faible niveau de revenu, ils se disent néanmoins satisfaits de leurs revenus (72\%). Alors qu'ils sont marginalisés par la société, non reconnus comme groupe ayant leur propre culture, ils se disent pourtant satisfaits de la sensibilisation de la population à l'endroit des personnes sourdes (72\%).

Ce niveau d'attente réduite confirme leur exclusion qui, par ailleurs, est intériorisée par les Sourds eux-mêmes. Ils n'attendent rien de l'extérieur, si ce n'est de leur famille ou de leurs amis immédiats. Ce constat permet de mieux saisir l'immense tâche qui attend les dirigeants de la communauté Sourde dans les années à venir. Ils devront lutter sur deux fronts. En premier lieu, auprès 
"La première tâche qui attend les dirigeants de la communauté

Sourde, de pair avec les différents ministères provinciaux, est de mousser la publicité des services existants dont peuvent se prévaloir les Sourds.» des instances gouvernementales, ils devront se faire les défenseurs des droits des Sourds en vue d'améliorer leurs conditions de vie. En second lieu, ils devront travailler auprès des Sourds pour faire émerger une conscience de leur valeur et ainsi forger cette culture Sourde qu'ils appellent de leurs vœux.

La première tâche qui attend les dirigeants de la communauté Sourde, de pair avec les différents ministères provinciaux, est de mousser la publicité des services existants dont peuvent se prévaloir les Sourds. En effet, notre enquête montre que seulement $36 \%$ des répondants $(n=45)$ connaissent le service d'appui d'un travailleur social offert aux Sourds par les bureaux régionaux du ministère des Services sociaux et communautaires de l'Ontario (M.S.S.C.O.). Le même constat se répète pour le service de formation de la main d'œuvre, où $30 \%$ des répondants disent connaitre ce service, pour le service d'assistance technique (28\%) ou pour le service de consultation psychologique (9\%).

Aussi, ne faut-il pas s'étonner de constater que seulement 11 répondants aient déjà utilisé un ou plusieurs services.

Auprès de ces services, la plupart des Sourds communique seul leur demande, par une combinaison de signes, de lecture labiale et de français parlé, mais il arrive que certains aient recours à un membre de la famille ou un ami servant d'interprète. Cette réalité ne devrait pas surprendre puisque les Sourds ne sont pas muets et ils ont appris, par la méthode oraliste, à exprimer leurs besoins, même si cela comporte des difficultés. C'est uniquement lorsque la difficulté devient grande que les Sourds nécessitent une aide externe (parent, ami, etc.). C'est notamment le cas lorsqu'ils ont à rencontrer un médecin en cabinet privé.

Là plus qu'ailleurs, ils utilisent dans une proportion importante les services d'un interprète, soit dans $50 \%$ des cas (23 sur 46). Il s'agit principalement d'un membre de la famille (conjoint, conjointe, parent, etc.) dans près de $69 \%$ des cas. Plus rarement, les Sourds utilisent les services d'interprétation professionnelle (de la SCO, d'étudiant en interprétation LSQ ou d'interprète venant du Québec), soit dans $22 \%$ des cas. Enfin, $9 \%$ des Sourds emploient un ami ou une autre personne comme interprète. $\mathrm{Si}$ l'on privilégie les membres de la famille à l'interprète professionnel, 
«Si l'on privilégie les membres de la famille à l'interprète professionnel, c'est souvent en raison $d u$ peu de disponibilité de ce dernier. Pour obtenir le service d'interprète, il faut prévoir son rendezvous, organiser un horaire qui convient au médecin et à l'interprète..." c'est souvent en raison du peu de disponibilité de ce dernier. Pour obtenir le service d'interprète, il faut prévoir son rendezvous, organiser un horaire qui convient au médecin et à l'interprète, comme le mentionnaient certains Sourds lors du prétest. Or, ces complications sont réduites lorsqu'on fait appel à un membre de la famille.

Si la plupart des Sourds mentionnaient être à l'aise en présence de leur interprète, leur surdité constitue une barrière importante pour communiquer leur malaise. Fait significatif, si 20 Sourds sur 45 affirment avoir eu de la difficulté à communiquer leur maladie lors d'une rencontre chez un médecin (44\%), ce pourcentage croît de façon importante en milieu hospitalier où $78 \%$ des Sourds affirment avoir eu semblables difficultés.

Dans le milieu hospitalier, les Sourds s'expriment seuls généralement $(52 \%, n=23)$. Lorsqu'ils requièrent les services d'un interprète, celui est principalement un membre de la famille (30 $\%, \mathrm{n}=23)$ ou un ami $(17 \%, \mathrm{n}=23)$. Aussi étonnant que cela puisse être, aucun Sourd n'a eu recours à un interprète professionnel en milieu hospitalier. Une double raison explique cette situation. D'une part, la communication avec le médecin traitant et le personnel infirmier est souvent spontanée et imprévisible, car elle se déroule au gré de l'horaire du personnel soignant. Or, il est impossible pour le Sourd de planifier une telle rencontre et de demander la présence d'un interprète professionnel au moment précis de la visite du médecin ou du personnel infirmier. Le second facteur, d'ordre plus institutionnel, tient au fait qu'il n'y a pas dans les hôpitaux de service d'interprétation en LSQ (ou en ASL), pas plus d'ailleurs qu'il n'y a de politique touchant la disponibilité de service d'interprétation sur appel pour les Sourds et avec lequel le médecin traitant pourrait communiquer lorsqu'il rencontre un Sourd.

D'aucuns diront que les services d'interprétation en langue des signes ne sont pas une demande formulée par la communauté Sourde dans son ensemble ${ }^{7}$. En effet, nous avons soumis aux Sourds une série de phrases portant sur les services d'interprétation en LSQ et nous leur avons demandé d'indiquer, sur une échelle de Likert, leur degré d'accord ou de désaccord. Les réponses sont 
Et s'il n'y avait qu'un développement à opérer pour satisfaire aux demandes des Sourds, ce serait justement d'établir un service d'interprétation en LSQ dans les diverses agences gouvernementales. regroupées de manière dichotomique (accord ou désaccord). Elles illustrent bien la position de la communauté Sourde.

Ainsi, 30 Sourds sur 43 (70 \%) considèrent qu'il y a un manque de services d'interprétation en langues des signes, ASL ou LSQ, dans sa région, alors que $7 \%$ des Sourds sont en désaccord avec une telle affirmation. Quant à la nécessité d'obtenir des services d'interprétation en LSQ, les Sourds sont presque unanimes puisque $86 \%$ des Sourds les jugent nécessaires. Près de $60 \%$ des Sourds sont d'accords pour dire qu'il n'est pas toujours possible d'obtenir les services d'un interprète.

Quant au besoin d'un interprète, il semble y avoir un large écart entre ce qui est du domaine communautaire ou privé et ce qui est du domaine étatique. Ce résultat se comprend aisément si l'on se rappelle que l'ensemble des Sourds sans emploi (15 sur 34) recevait des paiements de transferts pour subvenir à leurs besoins. Ainsi, le besoin d'interprète se fait particulièrement sentir pour les Sourds dans le domaine qui correspond à leur survie économique. Et s'il n'y avait qu'un développement à opérer pour satisfaire aux demandes des Sourds, ce serait justement d'établir un service d'interprétation en LSQ dans les diverses agences gouvernementales.

\section{Éducation et alphabétisme}

Nous avons ensuite cherché à connaitre le niveau d'éducation des Sourds. Actuellement, $31 \%(n=49)$ étudient à temps plein ou à temps partiel dans un établissement d'enseignement. De ce nombre, 6 étudient à temps plein et 9 étudient à temps partiel.

La majorité des Sourds ont terminé leurs études primaires (79\%, $n=43)$. Du même souffle, c'est dire que $21 \%(n=43)$ des Sourds ne les ont pas terminées. Des personnes qui ont terminé leurs études primaires, plus des deux tiers environ $(n=31)$ ont aussi terminé leurs études secondaires et $32 \%$ ne les ont pas terminées. 
«Il y a peu de services d'interprétation dans le Nord-est. Les gens $y$ sont dispersés sur un vaste territoire où la périphérie est mal desservie en termes de services éducatifs. Les Sourds ont peu de revenu et, puisqu'ils ont peu d'éducation, les Sourds ne savent pas où commencer.»
Si l'âge explique que plusieurs n'avaient pas terminé leurs études secondaires ${ }^{8}$, le fait que les Sourds aient dû évoluer dans des écoles secondaires françaises ou anglaises pour entendants n'a certainement pas contribué à leur réussite scolaire. En effet, 61\% $(\mathrm{n}=18)$ ont indiqué avoir terminé leurs études secondaires dans une école française pour entendants, 33\% dans une école française pour les Sourds et seulement 6\% a fréquenté une école anglaise pour Sourds. Sur les 21 Sourds ayant complété leurs études secondaires, 10 ont poursuivi au niveau collégial et universitaire, soit $48 \%$.

$\mathrm{Si}$ on considère qu'une personne n'ayant pas terminé sa neuvième année de secondaire est analphabète fonctionnelle, il y a parmi les Sourds environ $21 \%$ d'analphabètes ${ }^{9}$. Si l'on ajoute à ce nombre les Sourds n'ayant pas terminé leurs études secondaires, parce qu'ils possèdent un niveau d'éducation bien en deçà des exigences actuelles du marché du travail, on parvient à $44 \%(n=43)$ de Sourds ayant un urgent besoin de rattrapage scolaire, voire de cours d'alphabétisation.

Appelés à classer les facteurs qui empêcheraient un éventuel retour aux études par ordre d'importance, les Sourds ont indiqué que le manque de service d'interprétation était la principale barrière $(47 \%)$. Le second obstacle était la disponibilité de cours dans son milieu (33\%), suivi, en troisième lieu, de leur situation financière $(29 \%)$ et enfin, quatrièmement, du manque d'orientation pédagogique (28\%). Ces jugements correspondent à la réalité objective des Sourds francophones du Nord-est de l'Ontario. Il y a peu de services d'interprétation dans le Nord-est. Les gens y sont dispersés sur un vaste territoire où la périphérie est mal desservie en termes de services éducatifs. Les Sourds ont peu de revenu et, puisqu'ils ont peu d'éducation, les Sourds ne savent pas où commencer. Cela dit, les Sourds veulent s'intégrer et, en ce sens, l'éducation devient un moyen privilégié pour y parvenir. C'est pourquoi un nombre relativement important de Sourds se dit prêt à surmonter les obstacles et retourneraient à l'école s'ils en avaient l'occasion $(51 \%, \mathrm{n}=45)$. Il suffirait alors de les appuyer. 


\section{Malentendants et malentendantes}

Des 91 individus de notre échantillon global, 41 se déclarent malentendantes ou malentendants francophones $(98 \%)$ et un anglophone (2\%), soit 42 au total. Ce groupe de malentendants est composé de $65 \%$ d'hommes et $35 \%$ de femmes $(n=40)$. Dans le tableau 4, on remarque que ce groupe se répartit inégalement selon les catégories d'âge et le moment d'apparition de leur problème de surdité ou de déficience auditive.

Tableau 4

Données socio-démographiques sur les malentendants de

l'enquête

\begin{tabular}{|c|c|c|c|c|}
\hline $\begin{array}{l}\text { Sexe des } \\
\text { répondants } \\
(n=40)\end{array}$ & \multicolumn{2}{|c|}{$\begin{array}{l}\text { Âge des répondants } \\
(\mathrm{N}=42)\end{array}$} & \multicolumn{2}{|c|}{$\begin{array}{l}\text { Apparition de la surdité ou de la } \\
\text { déficience auditive }(\mathrm{N}=42)\end{array}$} \\
\hline $\begin{array}{ll}\text { Hommes } & 65 \% \\
\text { Femmes } & 35 \%\end{array}$ & $\begin{array}{l}\text { Moins de } 24 \text { ans } \\
25 \text { à } 44 \text { ans } \\
45 \text { à } 64 \text { ans } \\
65 \text { ans et plus }\end{array}$ & $\begin{array}{l}40 \% \\
10 \% \\
21 \% \\
29 \%\end{array}$ & $\begin{array}{l}\text { Avant l'âge de } 5 \text { ans } \\
\text { Entre } 5 \text { et } 14 \text { ans } \\
\text { Entre } 15 \text { et } 29 \text { ans } \\
40 \text { ans et plus }\end{array}$ & $\begin{array}{l}40,0 \% \\
7,2 \% \\
7,2 \% \\
38,0 \%\end{array}$ \\
\hline
\end{tabular}

Ces résultats interrogent à plus d'un titre. S'il est vrai que les personnes atteintes tardivement de déficience auditive, passé l'âge de 40 ans, ont tendance à se dire malentendantes plutôt que Sourdes (38\% chez les malentendants comparativement à seulement $11 \%$ chez les Sourds), l'inverse n'est pas vrai. Ainsi, les personnes atteintes de surdité en bas âge, moins de 14 ans, emploient indistinctement le terme malentendant et Sourd pour désigner leurs conditions et cela est surtout notable chez les jeunes.

L'explication réside dans le fait que l'épithète "malentendant» surgit, dans la langue française, au début des années soixante-dix, pour corriger la connotation péjorative associée au terme "sourd». Que les jeunes Sourds aient retenu ce qualificatif pour se désigner, correspond au changement d'attitude sociale envers la surdité, en 
"...l'épithète

«malentendant» surgit, dans la langue française, au début des années soixante-dix, pour corriger la connotation péjorative associée au terme "sourd». Que les jeunes Sourds aient retenu ce qualificatif pour se désigner, correspond au changement d'attitude sociale envers la surdité, en particulier d'un système scolaire cherchant à les intégrer davantage.» particulier d'un système scolaire cherchant à les intégrer davantage. Donc, le terme malentendant est affaire de génération et les jeunes Sourds ont davantage tendance à se désigner comme malentendant. Cependant, cette distinction peut aussi révéler une différence culturelle, une distinction d'appartenance entre ces deux groupes. Seule l'étude des autres caractéristiques permettront de dire si un tel clivage existe réellement.

Plus de la moitié du groupe malentendant est composé de gens mariés ou vivant en union libre $(54 \%, \mathrm{n}=41)$, les autres étant célibataires $(37 \%)$, veufs ou veuves $(7 \%)$ ou séparés $(2 \%)$. Les malentendants vivant en situation maritale ou en union libre ont un conjoint ou une conjointe francophone entendante $(86 \%$, $\mathrm{n}=22), 9 \%$ ont une ou un conjoint sourd francophone et un autre $5 \%$ ont une ou un conjoint malentendant francophone. Ce fait differe énormément par rapport au groupe Sourd. En effet, alors que seulement $27 \%$ des Sourds étaient mariés à des entendants francophones ou anglophones, ce pourcentage triple dans le cas des malentendants. Que les malentendants aient une ou un conjoint entendant ne surprend pas? L'âge est un facteur important. En effet, beaucoup de malentendants (38\%), susceptibles d'être mariés, ont vu apparaitre leur déficience auditive après l'âge de 40 ans, ce qui suppose leur participation antérieure au monde des entendants. Cette participation pourrait s'étendre aussi aux jeunes qui évoluent quotidiennement dans un monde d'entendants, leurs parents étant entendants eux-mêmes. Cela signifie que les malentendants participent davantage à la culture entendante que ne le font les Sourds. Ce fait, en apparence anodin, illustre que le sentiment d'appartenance à la culture Sourde, à moins d'avoir des parents qui y soient sensibles, se manifeste surtout à l'âge adulte, lorsque les Sourds prennent une certaine distance et gagnent en autonomie par rapport à leurs familles. Là s'installent alors les affinités avec d'autres Sourds, partageant la même langue (LSQ) et une même identité. Mais revenons aux malentendants.

Le tableau 5 montre quelques caractéristiques des malentendants quant à leur appartenance à la culture francophone. 
Tableau 5

\section{Caractéristiques des malentendants selon la langue parentale et la langue d'usage}

\begin{tabular}{|c|c|c|c|c|c|c|c|c|c|}
\hline \multicolumn{2}{|c|}{$\begin{array}{c}\text { Langue } \\
\text { maternelle }\end{array}$} & \multicolumn{2}{|c|}{ Langue paternelle } & \multicolumn{2}{|c|}{$\begin{array}{c}\text { Caractéristiques } \\
\text { du père }\end{array}$} & \multicolumn{2}{|c|}{$\begin{array}{l}\text { Caractéristiques } \\
\text { de la mère }\end{array}$} & \multicolumn{2}{|c|}{ Langue d'usage } \\
\hline Français & $91 \%$ & Français & $93 \%$ & Entendant & $92 \%$ & Entendante & $87 \%$ & Français & $94 \%$ \\
\hline LSQ & $4 \%$ & Anglais & $5 \%$ & Sourd & $3 \%$ & Sourde & $3 \%$ & LSQ & $3 \%$ \\
\hline Autres & $5 \%$ & LSQ & $2 \%$ & Malentendant & $5 \%$ & Malentendante & $0 \%$ & Autres & $3 \%$ \\
\hline
\end{tabular}

Du tableau 5 , on peut dire que la participation des malentendants à une culture entendante est clairement manifeste au niveau de la langue d'usage. En effet, on notera la quasi-absence de la LSQ comme langue d'usage. Cette absence signale que le malentendant adhère davantage au monde des entendants francophones que ne le font les Sourds. Et cette adhésion passe par la langue, soit dans le cas des malentendants, par le français, soit dans le cas des Sourds, par la LSQ.

D'ailleurs, lorsqu'on demande aux malentendants de dire à quelle culture ils s'identifient, $69 \%$ diront qu'ils s'identifient à la culture francophone entendante $(n=29)$, alors que le nombre de malentendants qui s'identifie à la culture sourde francophone est de $24 \%(n=29)$. Un malentendant s'identifie respectivement à la culture entendante anglophone $(3,5 \%)$ et mentionne appartenir à la culture malentendante $(3,5 \%)$.

«Parmi le groupe de malentendants, l'emploi d'un interprète devient superflu. Cela est surtout vrai des interprètes professionnels auxquels on n'a jamais recours.»
Parmi le groupe de malentendants, l'emploi d'un interprète devient superflu. Cela est surtout vrai des interprètes professionnels auxquels on n'a jamais recours. À la limite, on fera intervenir comme interprète un membre de la famille dans $26 \%(n=19)$ ou un ami dans $15 \%$. Pourtant, ces cas de figures demeurent marginaux. Le groupe de malentendants maitrise le français parlé. Cela n'étonne donc pas de voir que les moyens employés par les Sourds au niveau communicationnel ne leur conviennent pas. Dans tous les cas, les malentendants manifestent qu'ils n'utilisent guère ces moyens. Les malentendants emploieront, à l'occasion, une combinaison de signes, de lecture labiale et de français pour 
être compris, à l'instar des Sourds chez qui ce mode d'expression est dominant.

Comme le montre le tableau 6, le groupe de malentendants semble davantage intégré au monde du travail que celui des Sourds, $\mathrm{du}$ moins si nous nous fions au nombre de sans travail. Toutefois, cette affirmation doit être nuancée en raison de la faiblesse des nombres dans la distribution des occupations, des domaines d'emploi et des types de paiements de transferts reçus par les malentendants.

Tableau 6

Occupations, domaines d'emploi et paiements de transferts des personnes malentendantes

\begin{tabular}{|l|lr|ll|}
\hline $\begin{array}{l}\text { Occupation } \\
\text { (N=1710 ) }\end{array}$ & $\begin{array}{l}\text { Domaine d'emploi des } \\
\text { malentendants (N=14) }\end{array}$ & $\begin{array}{l}\text { Paiements de transferts } \\
\text { reçus par les } \\
\text { malentendants sans }\end{array}$ \\
emploi (N=13)
\end{tabular}

D'ailleurs, la faiblesse des revenus des malentendants ne semble pas témoignée de cette meilleure intégration au marché du travail. Cela dit, il faut aussi souligner que bon nombre des malentendants sont à la retraite, car ils ont atteint l'âge de 65 ans et plus (12 répondants sur 42). Par ailleurs, plusieurs sont aux études. Ces éléments peuvent contribuer à la faiblesse générale de leur revenu, comme le montre le tableau 7. 
Tableau 7

\section{Revenu des personnes malentendantes et de la conjointe ou du conjoint}

\begin{tabular}{|c|c|c|c|}
\hline \multicolumn{2}{|c|}{ Revenu des malentendants $(\mathrm{N}=34)$} & \multicolumn{2}{|c|}{ Revenu de la ou du conjoint $(\mathrm{N}=19)$} \\
\hline $\begin{array}{l}\text { Moins de } 10000 \$ \\
10000 \$ \text { à } 19999 \$ \\
20000 \$ \text { à } 39999 \$ \\
40000 \$ \text { et plus }\end{array}$ & $\begin{array}{l}47,1 \% \\
17,6 \% \\
29,4 \% \\
5,9 \%\end{array}$ & $\begin{array}{l}\text { Moins de } 10000 \$ \\
10000 \$ \text { à } 19999 \$ \\
20000 \$ \text { à } 39999 \$ \\
40000 \text { \$ et plus }\end{array}$ & $\begin{array}{l}47,1 \% \\
17,6 \% \\
29,4 \% \\
5,9 \%\end{array}$ \\
\hline
\end{tabular}

\section{Satisfactions et insatisfactions}

Pour les malentendants, l'insatisfaction manifestée envers les services gouvernementaux ne s'appuie pas réellement sur une bonne connaissance. En effet, très peu de malentendants connaissent les services offerts aux Sourds par le ministère des Services sociaux et communautaires de l'Ontario. Seulement 18 $\%$ des 40 malentendants connaissent le service d'appui d'un travailleur social et $5 \%$ celui de la formation de la main d'œuvre. Si le service des appareils techniques est le service le plus connu, il n'empêche que seulement $19 \%$ des malentendants affirment le connaitre. Fait intéressant à souligner, le service le plus connu des malentendants (l'aide aux appareils techniques) est également le plus employé. Que le service d'aide aux appareils techniques soit le plus connu et le plus utilisé par les malentendants ne surprend pas? La prothèse auditive devient, à leurs yeux, le symbole de leur participation au monde des entendants et marque la distance par rapport aux Sourds de naissance et de culture.

Les malentendants, à l'instar des Sourds, confirment le manque de services d'interprétation en langue des signes dans leur région (78\%) et, plus particulièrement, un manque de service 
d'interprétation en LSQ (74\%). Ils conçoivent également combien ces services d'interprétation en LSQ sont nécessaires pour les Sourds $(90 \%)$ et qu'il n'est pas toujours possible d'obtenir les services d'un interprète (82\%). Pourtant, cette conscience d'un manque de services d'interprétation en LSQ ne se manifesterait pas par un usage plus grand dans l'univers communautaire ou privé (famille, commerce, etc.). Le seul endroit où ce besoin de services d'interprétation en LSQ est véritablement senti comme essentiel, c'est dans les rapports avec les bureaucraties étatique ou autres (avocat, médecin, travailleur social, etc.). Là, $56 \%$ des malentendants souhaiteraient la présence d'un interprète.

Nous n'insisterons pas trop sur la langue apprise à la maison ou à l'école, de même que sur la langue d'usage à la maison, au travail ou dans les activités sociales chez les malentendants. Dans tous les cas, le français parlé arrive bon premier et est utilisé par plus de $85 \%$ des malentendants. En second lieu vient l'anglais parlé. Peu importe le domaine (maison, travail, activités sociales), la LSQ et l'ASL ne sont pratiquement pas employés par les malentendants. Il s'agit toujours d'une minorité.

\section{Éducation et alphabétisme}

"La situation scolaire des malentendants est similaire à celle des Sourds.»
La situation scolaire des malentendants est similaire à celle des Sourds. En effet, sur les 40 malentendants qui ont répondu, 24 seulement ont terminé leurs études primaires $(60 \%)$ et 16 ne les ont pas terminées. Cette situation s'explique par la distribution des personnes selon les catégories d'âges chez les malentendants. Il y avait un nombre considérable de personnes âgées à un pôle de la distribution et à l'autre pôle, de très jeunes gens ${ }^{11}$.

Des 24 malentendants ayant poursuivi des études secondaires, $71 \%$ ont terminé leurs études secondaires. Sur ces 17 personnes, 15 ont poursuivi au niveau collégial (9)ou universitaire (6). Il semble donc que les malentendants ont moins tendance que les Sourds à abandonner le système scolaire après leurs études 
"...les malentendants sont nettement plus ouverts à la méthode d'enseignement oraliste (lire sur les lèvres, et apprendre à produire les sons ou les mots comme le ferait un entendant) que ne le sont les Sourds qui cherchent à développer et à faire reconnaître leur propre langue (LSQ) et leur propre culture.» secondaires. Ce taux était de $48 \%$ chez les Sourds.

Bien que $40 \%$ des malentendants puissent être considérés comme des analphabètes fonctionnels (moins de 9 années de scolarité complétée), ils sont peu nombreux à vouloir suivre des cours d'alphabétisation. Les malentendants âgés ne voient guère l'utilité d'entreprendre de telles études à ce moment-ci. De fait, seulement $26 \%(n=35)$ seraient intéressés ou très intéressés à suivre des cours pour apprendre à lire et à écrire.

Quant à savoir si une grande importance a été accordée à la méthode oraliste durant leurs études, $67 \%$ des malentendants $(n=18)$ sont plutôt d'accord avec ce jugement, tandis $33 \%$ sont plutôt en désaccord. C'est dire que les malentendants sont nettement plus ouverts à la méthode d'enseignement oraliste (lire sur les lèvres, et apprendre à produire les sons ou les mots comme le ferait un entendant) que ne le sont les Sourds qui cherchent à développer et à faire reconnaître leur propre langue (LSQ) et leur propre culture. En effet, chez les Sourds $(n=42), 52 \%$ envisageaient défavorablement ou très défavorablement l'enseignement de la langue selon la méthode oraliste.

\section{Conclusion}

À la lumière des résultats obtenus, on ne peut pas douter de l'existence d'une culture sourde franco-ontarienne. La LSQ (langue des signes québécoise) est la langue d'usage de cette communauté. Cette culture est aussi caractérisée par une forte endogamie, puisque les Sourds se marient généralement entre eux. Or, le sentiment d'appartenance à la culture Sourde, à moins d'avoir des parents qui y soient sensibles, se manifeste surtout à l'adolescence ou à l'âge adulte, lorsque les Sourds prennent une certaine distance et gagnent en autonomie par rapport à leurs familles. Entre-temps, la surdité force la solidarité familiale en développant une interdépendance beaucoup plus grande entre les membres de la famille. Ceux-ci sont alors appelés à agir 
d'intermédiaire entre l'univers des Sourds et le monde des entendants. Par ailleurs, notre étude montre que la différence entre les Sourds et les malentendants se situe justement dans leur degré d'intégration aux mondes des entendants. Au-delà de l'étiquette et de l'âge à laquelle survient la surdité, un malentendant est une personne mieux intégrée au monde des entendants et la prothèse auditive en est le symbole.

Par ailleurs, la culture Sourde est aussi une culture de pauvreté en raison des faibles revenus de sa population. Elle est aussi une culture de sans travail, car près de la moitié des Sourds est sans emploi. Par conséquent, une forte proportion des sans emploi reçoit des paiements de transferts des gouvernements. Elle est aussi une communauté qui nécessite un effort soutenu en ce qui concerne son éducation, puisque $44 \%$ des Sourds n'ont pas terminé leurs études secondaires.

Devant les tendances lourdes qui affectent cette communauté, il faut être sensible à leurs revendications. Aussi, faut-il favoriser l'accès aux interprètes en LSQ (langue des signes québécois) et fournir également plus de ressources financières pour l'utilisation des services en LSQ. La présence de services d'un interprète est souhaitable et souhaitée dans les domaines touchant les relations avec le gouvernement, tant par les Sourds que par les malentendants. Certes, la famille compense la plupart du temps le manque d'interprètes professionnels. Mais, ce palliatif est loin d'être un gage d'autonomie. Qui plus est, les services d'interprétation en LSQ deviennent un support essentiel, si on entend accroitre la formation scolaire des Sourds et des malentendants. Par ailleurs, il faut aussi faire davantage connaître à la population Sourde et malentendante, les différents services offerts par les divers organismes gouvernementaux et para-gouvernementaux.

\section{Bibliographie ${ }^{12}$}

ASSOCIATION DES SOURDS ET SOURDES FRANCOPHONES DE SUDBURY (1996). Une culture qui s'entend, Sudbury.

GARCEAU, MARIE-LUCE (1995). Les Franco-Ontariennes de 45 à 64 ans: analyse de leurs conditions 
de vie, Montréal, UQAM, thèse de doctorat.

LANE, HARLAN (1993). «L'oppression dans les relations entre les professionnels entendants et les sourds de culture». Nouvelles pratiques sociales, vol.6, no1, 41-56.

MOTTEZ, BERNARD (1985). «Aspects de la culture sourde», Santé mentale: «L'œil écoute», Paris, no. 85, 13-16.

PROULX,JEAN-PIERRE (1994). «L'analphabétisme» in Traité des problèmes sociaux, Montréal, Institut québécois de recherche sur la culture.

RICHER-ST-LOUIS, MURIELLE. (1998). Mémoires présentés dans le cadre de la consultation ODA du Ministère des affaires civiques, de la culture et des loisirs, Gouvernement de l'Ontario, 11 juillet, texte ronéotypé, 1-6.

SOCIÉTÉ CANADIENNE DE L'OUÏE, BUREAU RÉGIONAL D’OTTAWA (1990). Étude sur les besoins des francophones sourds et malentendants de la région de l'Est de l'Ontario, Ottawa, Société canadienne de l'Ouïe.

STATISTIQUES CANADA (1989). Enquête sur la santé et les limitations d'activités, données infra provinciales, Ontario, Catalogue 82-608, Ottawa, Ministère des Approvisionnements et services.

STATISTIQUES CANADA (1991). Enquête sur la santé et les limitations d'activités. Tableaux d'appoints, Ontario, Ottawa, Ministère des Approvisionnements et services.

VALLIÈRES, MICHELINE ET AL. (1993). «Briser le silence entourant le monde des Sourds». Nouvelles pratiques sociales, vol. 6, no 1,34-39.

\section{Notes}

1. Le présent article s'inspire largement d'un rapport de recherche dont je suis l'auteur et qui fut présenté à l'Association des Sourds et des Sourdes francophones de Sudbury, intitulé «Une culture qui s'entend», en février 1996. Je tiens à remercier les personnes qui ont participé à la recherche. Mes remerciements vont d'abord à M. Donald Dennie de l'Institut franco-ontarien, un collègue, mais surtout un complice. Par ailleurs, j'aimerais remercier Roger St-Louis et son fils Joël qui ont initié cette recherche et, enfin, toute l'équipe de collecte de données sur le terrain:James Tregonning, Paul Groulx, Éric Grenier et Linda Garand.

2. Par convention, on écrit «Sourd» avec la majuscule pour se référer aux personnes revendiquant leur appartenance à la culture sourde et «sourd» avec une minuscule pour référer aux aspects physiologiques de la surdité (Vallières et al. 1994:34).

3. Nous pensons ici aux multiples appels aux organismes francophones, aux audiologistes, aux paroisses desservant les 
Sourds et les malentendants ainsi qu'à la mise à profit de la compagnie Bell Canada et d'une tournée dans le Nord-est de l'Ontario qui a permis de maximiser le repérage des Sourds (voir à ce sujet, Association des Sourdes et des Sourds francophones 1996).

4. Voir à ce sujet le texte d'André Tremblay (1991), Sondages: histoire, pratique et analyse, Gaëtan Morin éditeur, pages 173 et ss.

5. Nous avons exclu de la catégorie sans emploi, les personnes de 65 ans et plus et les jeunes de moins de 16 ans. Au total, il s'agit de 11 personnes. De plus, il y avait 4 données manquantes à la question de l'occupation.

6. Ce chiffre a été établi par le Conseil national du bien-être social (1994), Profil de la pauvreté, 1992, Ottawa, Ministère des Approvisionnements et Services Canada.

7. Voir le document de Murielle St-Louis, de l'Association ontarienne des Sourds et des Sourdes francophones, présenté dans le cadre de la consultation menée par le gouvernement ontarien sur un projet de loi "Ontarians with Disabilities Act», juillet 1998.

8. De fait, 15 personnes sur les 19 Sourds n'ayant pas complété leurs études primaires et secondaires sont âgées de plus de 45 ans, au moment de l'enquête. De plus, 3 Sourds sont actuellement inscrits dans une école secondaire.

9. Proulx, Jean-Pierre (1994). «L'analphabétisme» in Traité des problèmes sociaux, Montréal, Institut québécois de recherche sur la culture.

10. Nous avons exclu de la catégorie sans emploi, les personnes âgées de 65 ans et plus (12 répondants) et les moins de 16 ans (9 répondants). Au total, il s'agit de 21 personnes. De plus, il y avait 4 données manquantes à la question de l'occupation.

11. De fait, 11 personnes sur 19 n'ayant pas complété leurs études primaires et secondaires sont âgées de 45 et plus. De plus, 5 jeunes de moins de 16 ans n'ont pas complété leurs études primaires et secondaires et sont présentement aux études.Ainsi, le niveau éducationnel atteint est un phénomène de génération.

12. Pour toutes questions concernant la documentation sur la surdité en français, on consultera à profit la revue Nouvelles pratiques sociales, volume 6, numéro 1, printemps 1993. 